\section{MODERATE AORTIC DILATATION SHOULD NOT BE IGNORED AT PRIMARILY NONAORTIC CARDIAC SURGERY}

To the Editor:

We read with interest the article by Idrees and colleagues ${ }^{1}$ addressing the important issue of whether prophylactic ascending aortic replacement confers additional risk when performed as a concomitant procedure to primarily nonaortic cardiac surgery. Analyzing 647 patients submitted to a combined cardioaortic procedure who were propensitymatched to a non-aortic surgery cohort, they showed no incremental risk for in-hospital stroke $(1.4 \%$ vs $1.1 \%)$ or mortality $(0.93 \%$ vs $0.46 \%)$.

Operative risk stratification is largely centered on scoring systems such as the Society of Thoracic Surgeons or European System for Cardiac Operative Risk Evaluation (EuroSCORE). These algorithms for mortality risk prediction are validated in general cardiac populations, although EuroSCORE overestimates risk for combined aortic procedures and underestimates it in isolated major aortic surgery. Furthermore, the most recent iteration of EuroSCORE incorporates only 1 parameter accounting for surgery on the thoracic aorta despite its variations and complexities. A reliable risk calculator dedicated to aortic surgery, integrating essential characteristics such as the segment of the aorta involved, acute aortic syndrome, connective tissue disease, valve morphology, and genetic data is now needed.

Contemporary guidelines ${ }^{2}$ advocate preventative aortic replacement at diameters of 4.5 to $5.5 \mathrm{~cm}$, dependent on risk factors, valvular function, concomitant procedures, and surgeon experience, to mitigate the threat of aortic dissection or rupture associated with progressive aneurysmal dilatation. However, diameter is not the sole determinant of aortic risk, and mounting evidence indicates that adverse events may develop acutely at "benign" diameters significantly smaller than those routinely considered for prophylactic replacement. Even mild ascending aortic dilatation of 4.0 to $4.4 \mathrm{~cm}$ represents a striking 89 -fold increased risk of dissection. ${ }^{3}$ The risks of operating at smaller aortic diameters nevertheless have to be balanced against those

\footnotetext{
The Editor welcomes submissions for possible publication in the Letters to the Editor section that consist of commentary on an article published in the Journal or other relevant issues. Authors should: $\bullet$ Include no more than 500 words of text, three authors, and five references. • Type with double-spacing. • See http://jtcs.ctsnetjournals.org/ misc/ifora.shtml for detailed submission instructions. • Submit the letter electronically via jtcvs.editorialmanager.com. Letters commenting on an article published in the JTCVS will be considered if they are received within 6 weeks of the time the article was published. Authors of the article being commented on will be given an opportunity of offer a timely response ( 2 weeks) to the letter. Authors of letters will be notified that the letter has been received. Unpublished letters cannot be returned.
}

of ongoing surveillance, during which patients may still be exposed to potentially life-threatening aortic complications.

Indexed measurements of aortic dimension can facilitate the identification of these smaller aortic aneurysms that do not satisfy size criteria for prophylactic resection, yet are still susceptible to aortic dissection. Svensson and Khitin ${ }^{4}$ previously proposed that an abnormal ratio of the cross-sectional aortic area indexed to patient height $>10 \mathrm{~cm}^{2} / \mathrm{m}$ reflects an increased dissection/ rupture risk. ${ }^{4}$ In an earlier analysis by our group, we showed that $69.5 \%$ of aortic root/ascending aortic aneurysms had an abnormal ratio alongside aortic diameters $<5.5 \mathrm{~cm}^{5}$

In the study by Idrees and colleagues, we would similarly expect a significant proportion of those patients undergoing proximal aortic replacement, who had a mean aortic diameter of $4.9 \pm 0.95 \mathrm{~cm}$, to possess an abnormally increased ratio. $37 \%$ of these patients had bicuspid aortopathy; perhaps a more aggressive stance towards aortic replacement is necessary in this vulnerable group with up to 9-fold magnified dissection/rupture risks.

We concur with Idrees and colleagues' that concomitant ascending aortic replacement at experienced, high-volume aortic centers is safe and achieves good outcomes. As cardiac surgeons, we are visually accustomed to and comfortable managing large aortas. We must be careful not to dismiss the opportunity for earlier intervention in subthreshold aortic aneurysms, especially when associated with bicuspid aortic valves. This strategy could save some patients from redo surgery for rapid aneurysmal expansion or acute dissection.

\section{Metesh Acharya, MRCS Marjan Jahangiri, FRCS (CTh) Department of Cardiothoracic Surgery St George's Hospital London, United Kingdom}

\section{References}

1. Idrees JJ, Roselli EE, Blackstone EH, Lowry AM, Soltesz EG, Johnston DR, et al. Risk of adding prophylactic aorta replacement to a cardiac operation. J Thorac Cardiovasc Surg. 2020;159:1669-78.

2. Hiratzka LF, Bakris GL, Beckman JA, Bersin RM, Carr VF, Casey DE Jr, et al 2010 ACCF/AHA/AATS/ACR/ASA/SCA/SCAI/SIR/STS/SVM guidelines for the diagnosis and management of patients with thoracic aortic disease: a report of the American College of Cardiology Foundation/American Heart Association task force on practical guidelines, American Association for Thoracic Surgery, American College of Radiology, American Stroke Association, Society of Cardiovascular Anesthesiologists, Society for Cardiovascular Angiography and Interventions, Society of Interventional Radiology, Society of Thoracic Surgeons, and Society for Vascular Medicine. Circulation. 2010;121:e266-369.

3. Paruchuri V, Salhab KF, Kuzmik G, Gubernikoff G, Fang H, Rizzo JA, et al. Aortic size distribution in the general population: explaining the size paradox in aortic dissection. Cardiology. 2015;131:265-72.

4. Svensson LG, Khitin L. Aortic cross-sectional area/height ratio and timing of aortic surgery in asymptomatic patients with Marfan syndrome. J Thorac Cardiovasc Surg. 2002;123:360-1. 
The authors reported no conflicts of interest.

The Journal policy requires editors and reviewers to disclose conflicts of interest and to decline handling or reviewing manuscripts for which they may have a conflict of interest. The editors and reviewers of this article have no conflicts of interest.

5. Acharya MN, Youssefi P, Soppa G, Valencia O, Nowell J, Kanagasabay R, et al. Analysis of aortic area/height ratio in patients with thoracic aortic aneurysm and type A dissection. Eur J Cardiothorac Surg. 2018;54:696-701.

https://doi.org/10.1016/j.jtcvs.2020.08.007

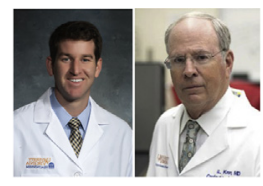

\section{REPLY: CONCOMITANT AORTIC REPLACEMENT: HOW PROACTIVE SHOULD WE BE? Reply to the Editor:}

There is a growing body of literature demonstrating that concomitant aortic replacement at the time of elective cardiac surgery can be safely performed. In a recent article by Idrees and colleagues ${ }^{1}$ in the Journal, they compare a cohort undergoing combined cardiac and aortic procedures with those undergoing cardiac surgery alone and found no difference in rate of in hospital stroke $(1.4 \%$ vs $1.1 \%)$ or mortality $(0.93 \%$ vs $0.46 \%)$. Current guidelines recommend concomitant replacement of the ascending aorta with dilation measuring greater than 4.5 to $5.5 \mathrm{~cm}$ diameter depending on risk factors. ${ }^{2}$ The majority of patients in the study by Idrees and colleagues were within this range (mean $4.8 \mathrm{~cm})^{1}$

In a recent letter to the editor, Acharya and Jahangiri ${ }^{3}$ suggest that "diameter is not the sole determinant of aortic risk" and that by using only this lens we could be missing an opportunity to concomitantly intervene on smaller "benign" aneurysms that may ultimately progress requiring future correction or result in dissection. For example, they present findings from Paruchuri and colleagues ${ }^{4}$ that even mild proximal aortic dilation $(4.0-4.4 \mathrm{~cm})$ carries an 89 -fold increased risk of dissection. We read this letter with interest as it brings up an important question-how proactive should we be? Certainly, diameter is not the only determinant of risk. The likelihood of disease progression incorporates multiple factors including, but not limited to, valvular anatomy, aneurysm location, presence of genetic disorder, and presence of connective tissue disease.

We agree that consideration of these factors is important for surgical decision-making. This is reflected, to some degree, in current guidelines, as replacement is indicated for aneurysms larger than 4 to $5 \mathrm{~cm}$ in the presence of a bicuspid aortic valve or one of several genetically mediated disorders, including Marfan syndrome, vascular Ehlers-Danlos syndrome, or Turner syndrome. ${ }^{2}$ However, as described in the letter, the risk of operating must be balanced against the risk of continued observation. In the absence of one of these factors, we feel that current indications for concomitant repair $(4.5-5.5 \mathrm{~cm}$ diameter) must apply. Although operative risk is not markedly increased, proximal aortic replacement at the time of elective cardiac surgery increases operative time and complexity, and this must be balanced against the risk of disease progression. While mild ascending aortic dilation (4.0-4.4 cm) carries an 89-fold increased risk of future dissection, dilation to $4.5 \mathrm{~cm}$ carries a stark 346-fold increase in risk. ${ }^{4}$ It is likely at this inflection point that the risk of continued observation outweighs the risk of operative intervention. While mean aortic dilation in the study by Idrees and colleagues was $4.8 \mathrm{~cm},{ }^{1}$ It would be interesting to evaluate outcomes within a smaller diameter $(<4.5 \mathrm{~cm})$ subgroup for comparison.

Acharya and Jahangiri ${ }^{3}$ propose an alternative strategy using an indexed measure of aortic dimension relative to patient height to identify aneurysms that may be at greater risk for dissection. Although there is emerging evidence that this measurement may aid in prediction, we feel that it should not supersede diameter-a parameter that has been rigorously studied for decades. Additional, diligent study of this indexed measurement is needed to understand its predictive power. At current, its primary utility in concomitant aortic replacement should be as an adjunct in surgical decision making within the constraints of accepted guidelines.

This letter brings to light important considerations for concurrent aortic replacement at the time of elective cardiac surgery. Namely, it raises the question if we should be more proactive in our approach. We agree with the authors that risk of disease progression is multifactorial and there is some opportunity for nuanced surgical decision making; however, we should proceed within current guidelines to optimize the benefit to risk ratio.

Nathan Haywood, $M D^{a}$

Irving L. Kron, $M D^{b}$

${ }^{a}$ Division of Thoracic and Cardiovascular Surgery

Department of Surgery

University of Virginia

Charlottesville, $\mathrm{Va}$

${ }^{b}$ Department of Surgery

University of Arizona-Tucson

Tucson, Ariz

\section{References}

1. Idrees JJ, Roselli EE, Blackstone EH, Lowry AM, Soltesz EG, Johnston DR, et al Risk of adding prophylactic aorta replacement to a cardiac operation. J Thorac Cardiovasc Surg. 2020;159:1669-78.e10. 